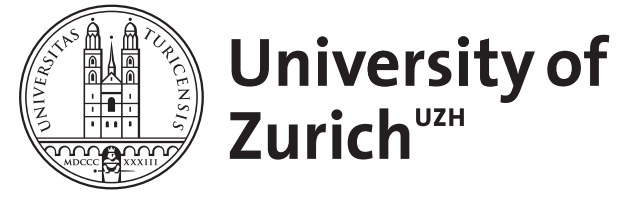

\title{
Gesetz und Weisheit im Pentateuch
}

\author{
Krüger, Thomas
}

DOI: https://doi.org/10.1515/9783110923988.1

Posted at the Zurich Open Repository and Archive, University of Zurich ZORA URL: https://doi.org/10.5167/uzh-69716

Book Section

Published Version

Originally published at:

Krüger, Thomas (2003). Gesetz und Weisheit im Pentateuch. In: Fischer, I; Rapp, U; Schiller, J. Auf den Spuren der Schriftgelehrten Weisen. Berlin: de Gruyter, 1-12.

DOI: https://doi.org/10.1515/9783110923988.1 
Ludger Schwienhorst-Schönberger

Vertritt Kohelet die Lehre vom absoluten Tod?

Zum Argumentationsgang von Koh 9,1-6

Ernst Haag

Weisheit und Heilsgeschichte. Das Dankgebet Daniels

in Daniel 2,20-23

\section{Pancratius C. Beentjes}

Tränen, Trauer, Totenklage. Eine kleine Studie über Ben Sira 38,16--23 ... 233

Nuria Calduch-Benages

Dreams and Folly in Sir 34(31), 1-8

\section{Alexander A. Di Lella}

Free Will in the Wisdom of Ben Sira 15:11-20. An Exegetical and

Theological Study

Heinz-Josef Fabry

Jesus Sirach und das Priestertum

Maurice Gilbert

Venez à mon école (Si 51,13-30)

Otto Kaiser

Göttliche Weisheit und menschliche Freiheit bei Ben Sira

\section{Josef M. Oesch}

Textdarstellungen in den hebräischen Sirachhandschriften

Ursula Rapp

Der gottesfürchtigen Frau ein guter Mann? Zur Lektüre der Aussagen

über gute und schlechte Ehefrauen im Sirachbuch

Georg Sauer

Ben Sira in Jerusalem und sein Enkel in Alexandria

Adrian Schenker

Erlässt Umkehr Schuld oder vermindert sie Strafe? Jesus Sirach

(Sir 17), Nabots Weinberg (1 Kön 21), Ezechiel (Ez 18), zugleich

ein Beitrag zum Verhältnis zwischen massoretischem Text und

Septuaginta in 1 Kön 21

Armin Schmitt

Enkomien in griechischer Literatur

Gerhard Bodendorfer

David, der weise Toragelehrte. Zur Funktion Davids

im babylonischen Talmud

Günter Stemberger

„Ich habe nichts Besseres für den Menschen gefunden

als Schweigen" (mAv 1,17) .

\section{Gesetz und Weisheit im Pentateuch}

Thomas Krüger

$I$.

Gesetz und Weisheit ${ }^{1}$ werden im Alten Testament vor allem an zwei relativ späten Stellen programmatisch miteinander identifiziert: bei Jesus Sirach (Kap. 24, hier besonders V. 23) und im apokryphen Baruchbuch (3,9-4,4, besonders 4,1). Johannes Marböck hat die hier entwickelten theologischen Konzeptionen, ihren zeitgeschichtlichen Kontext und ihre traditionsgeschichtlichen Voraussetzungen und Folgen mustergültig herausgearbeitet. ${ }^{2}$ Sir 24 konzipiert eine Verbindung von Weisheit und Gesetz, in der sich beide gegenseitig interpretieren und ergänzen. Die Weisheit verkörpert sich - zumindest für Israel - maßgeblich im mosaischen Gesetz. Zugleich wird aber auch das Gesetz als eine unausschöpfliche Weisheitslehre verstanden, die allen daran Interessierten offen steht.

„Vielleicht darf man so formulieren: fur Israel ist die Weisheit, mit allem, was darunter

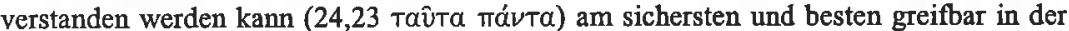
Torah vor den Völkern der Welt ist die Torah Israels Weisheit. Beide aber kommen vom Herrn, sind Weisen seines Wirkens und seiner Gegenwart in der Welt. ${ }^{43}$

Verglichen mit Sir 24 ist in Bar 3f. ,[d]er Horizont ... noch enger geworden. Bei Baruch gibt es keine von Gesetz und Israel unabhängige Weisheit mehr". ${ }^{4}$ Die im Gesetz verkörperte Weisheit besteht nach Bar 3f. in Handlungsanweisungen, die es zu befolgen gilt (und nicht in Einsichten, die zu

1 Vgl. Blenkinsopp, Joseph, Wisdom and Law in the Old Testament. The Ordering of Life in Israel and Early Judaism, OBS, Oxford ${ }^{2} 1995$.

2 Vgl. Marböck, Johannes, Weisheit im Wandel. Untersuchungen zur Weisheitstheologie bei Ben Sira, BZAW 272 , Berlin u.a. ${ }^{2} 1999$, 34ff.; ders., Gesetz und Weisheit. Zum Verständnis des Gesetzes bei Jesus Ben Sira, in: ders., Gottes Weisheit unter uns. Zur Theologie des Buches Sirach, HBS 6, Freiburg i.Br. u.a. 1995, 52-72, hier bes. 57ff.; ders., Gottes Weisheit unter uns. Sir 24 als Beitrag zur biblischen Theologie, in: ders., Gottes Weisheit, 73-87, hier bes. 85f.

3 Marböck, Weisheit (s. Anm. 2), $94 f$.

4 Marböck, Weisheit (s. Anm. 2) 57. - Whhrend in Sir 24 ,das Gesetz in die weiterreichende Weisheit einbezogen“ wird, gilt nach Bar 3f.: „Nichts anderes als das allein Israel gegebene Gesetz ist diese Weisheit; hier ist ihr einzig zugänglicher Ort" (Steck, Odil H., Das apokryphe Baruchbuch. Studien zu Rezeption und Konzentration „kanonischer“ Überlieferung, FRLANT 160, Göttingen 1993, 156, vgl. 116ff.). 
unerschöpflichem weiteren Nachdenken anregen). In den Differenzen zwischen diesen beiden Konzepten spiegeln sich unterschiedliche Erfahrungen und Deutungen der Auseinandersetzung mit hellenistischer Kultur und Weisheit vor und nach der Krise unter Antiochus IV. ${ }^{5}$ Darin und dahinter verweisen sie aber auch auf eine Reihe von theologischen Sachproblemen.

Versucht man, Gesetz und Weisheit idealtypisch einander gegenüberzustellen, so geht es beiden auf je verschiedene Weise darum, die menschliche Lebensführung zu bestimmen oder mindestens zu beeinflussen. Während ein Gesetz moralische und rechtliche Normen menschlichen Verhaltens verbindlich festlegt und unter Androhung von Sanktionen deren Befolgung fordert, ${ }^{6}$ will eine Weisheitslehre handlungsleitendes Orientierungswissen (über Tatsachen, Normen oder Werte) vermitteln und damit zu einer erfolgreichen Lebensführung befähigen. Das Gesetz verdankt seine Geltung der Autorität des Gesetzgebers und fordert unter Strafandrohung den Gehorsam derer, für die es gilt. Demgegenüber appelliert die Weisheit an die Einsicht des Menschen und beruft sich auf die Bewährung ihrer Ratschläge in der Lebenserfahrung (wobei der Akzent auf aktuellen Erfahrungen des Weisheitslehrers und/oder seiner Adressaten liegen kann oder auf den Erfahrungen früherer Generationen, also auf der Tradition). Während ein Gesetz in seinem Geltungsbereich von allen Menschen zu befolgen ist, sind nicht alle Menschen in gleichem Maße für Weisheitslehren empfänglich (es gibt „Weise“ und „Toren"). Andererseits wendet sich die Weisheit an alle einsichtsfähigen Menschen, unabhängig von ihrer ethnischen oder kulturellen Zugehörigkeit, während das Gesetz nur für eine begrenzte Gruppe Geltung beansprucht.

Mit dem Verhältnis von Weisheit und Gesetz stehen somit u.a. die folgenden Sachprobleme zur Diskussion: Welche Bedeutung haben kollektiv gültige Normen einerseits und individuelle Erfahrungen und Einsichten andererseits für die Lebensorientierung und Lebensführung des Menschen und wie sind beide Gesichtspunkte miteinander zu verbinden? In welchem Verhältnis stehen Tradition, göttliche Offenbarung und eigene Erfahrung, Einsicht und Erkenntnis zueinander? Und wie verhalten sich kulturspezifische (,israelitische“) Erfahrungen, Einsichten, Werte und Normen zu solchen, die kulturübergreifend (,interkulturell') geteilt werden? Johannes Marböck hat die Entwicklung dieser Diskussion in der alttestamentlichen und der spätisraelitischen Literatur im Vorfeld von Sirach und Baruch und im Anschluss

5 Zur Datierung von Sirach um 175 v.Chr. vgl. Marböck, Johannes, Das Buch Jesus Sirach in: Zenger, Erich u.a., Einleitung in das Alte Testament, KStTh 1,1, Stuttgart u.a. ${ }^{3} 1998$, 363-370, 367f., zur Datierung von Baruch (nicht lange) nach 164 v.Chr. siehe Steck, Baruchbuch (s. Anm. 4), $285 \mathrm{ff}$

6 Das gilt jedenfalls für Rechtsgesetze, die hier zunächst im Blick sind, mit Einschränkungen auch für moralische bzw. Sittengesetze. Anders verhält es sich bei sog. Seinsgesetzen, die hier ausgeklammert bleiben können. daran bereits sorgfältig nachgezeichnet. ${ }^{7}$ Im Folgenden soll das Augenmerk speziell auf einige Ansätze zur Diskussion dieser Fragen im Pentateuch gerichtet werden.

\section{$I I$}

Den deutlichsten Anknüpfungspunkt für eine Identifikation von Gesetz und Weisheit im Pentateuch bietet zweifellos Dtn $4,5-8,{ }^{8}$ wo Mose zu Israel spricht:

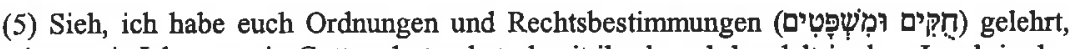
wie es mir Jahwe, mein Gott, geboten hat, damit ihr danach handelt in dem Land, in das ihr nun einzieht, um es in Besitz zu nehmen. (6) Haltet sie ein und handelt danach! Denr

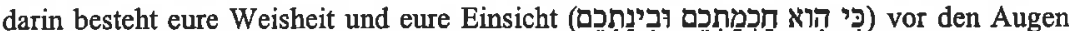
der Völker. Wenn sie all diese Ordnungen (םיקח) hören werden, dann werden sie sagen:

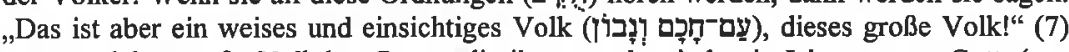
Denn welches große Volk hat Götter, die ihm so nahe sind, wie Jahwe, unser Gott, (uns nahe ist) wann immer wir zu ihm rufen? (8) Und welches große Volk hat Ordnungen und

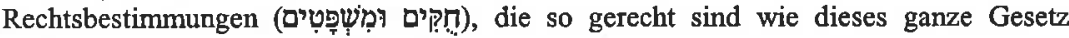
(กרָiภ), das ich euch heute vorlege?

Es ist nicht ganz klar, ob in V. 6 die „Ordnungen (und Rechtsbestimmungen)" des Gesetzes als "Weisheit und Einsicht" der Israeliten bezeichnet werden sollen oder deren Einhaltung durch Israel. ${ }^{9}$ Sachlich besteht zwischen beiden Deutungsmöglichkeiten allerdings kein großer Unterschied. Jedenfalls sind die Gesetzesbestimmungen ,gerecht" (V. 8), und deshalb ist es weise und vernünftig, sie zu befolgen. $\mathrm{Zu}$ dieser Einsicht müssen nach $\mathrm{Dtn} 4$ nicht nur die Israeliten kommen, sondern auch alle anderen Völker, die von der Thora Kenntnis erhalten. Das setzt voraus, dass Israel das Gesetz (entgegen der Forderung von Bar 4,3) den anderen Völkern nicht vorenthält, sondern es ihnen zur Kenntnis bringt (vgl. Sir 24,31ff.). Es setzt weiter voraus, dass die anderen Völker ebenso wie Israel im Stande sind, die Qualität des Gesetzes zu erkennen und insofern auch selbst über ein gewisses $\mathrm{Ma} ß$ an „Weisheit und Einsicht" verfügen. Allerdings haben die anderen Völker nach Dtn 4 nicht so gerechte Gesetze wie Israel. Das ist ein Hinweis darauf, dass sie zwar in der Lage sind einzusehen, dass das ihnen vorgelegte Gesetz Israels gerecht und vernünftig ist, nicht aber aus eigener Einsicht Gesetze von gleicher Qualität aufzustellen vermögen. Mit der Behauptung der Einsichtigkeit

7 Siehe oben Anm. 2.

8 Vgl dazu (neben den Kommentaren) Braulik, Georg, "Weisheit" im Buch Deuteronomium, in: Janowski, Bernd (Hg.), Weisheit außerhalb der kanonischen Weisheitsschriften, VWGTh 10, Gütersloh 1996, 39-69, 54ff.; ders., Weisheit, Gottesnăhe und Gesetz Zum Kerygma von Deuteronomium 4,5-8, in: ders., Studien zur Theologie des Deuteronomiums, SBAB 2, Stuttgart 1988, 53-93.

9 Vgl. Braulik, „Weisheit" (s. Anm. 8), 56f. 
und Vernünftigkeit des Gesetzes wird nach Dtn 4 seine Offenbarungsqualität demnach keineswegs in Frage gestellt.

Das Verhältnis von Weisheit und Gesetz wird so in Dtn 4 nochmals anders bestimmt als in Sir 24 und in Bar 3f.: Die besondere Weisheit Israels besteht darin, dass es gerechte Gesetze hat und diese befolgt. Das ist nach Ansicht von Dtn 4 auch anderen Völkern einsichtig, sobald sie das Gesetz zur Kenntnis nehmen. Um dies erkennen zu können, müssen die anderen Völker aber auch selbst - unabhängig von der Thora - über ein gewisses Maß an „Weisheit und Einsicht“ verfügen. Wenn Israels „Weisheit und Einsicht" in seiner exzellenten Rechtsordnung und Gesetzesbefolgung besteht, wird damit des Weiteren auch die Möglichkeit offen gelassen, dass andere Völker eine Israel überlegene "Weisheit und Einsicht" in anderen Bereichen (wie etwa der Kosmologie oder der Technologie) besitzen können. Nicht "die Weisheit" ist Israel im Gesetz mitgeteilt, sondern „Israels Weisheit“ unter den Völkern besteht in seiner Auszeichnung durch das (gerechte) Gesetz.

Dass auch die anderen Völker die Thora zur Kenntnis nehmen und ihre Qualität (an)erkennen, kommt im Pentateuch sonst nicht in den Blick. ${ }^{10}$ In Bezug auf Israel wird dagegen mehrfach darauf hingewiesen, dass das Gesetz nicht (nur) wegen der Autorität des Gesetzgebers zu befolgen ist, ${ }^{11}$ sondern (auch) aufgrund der Einsicht, dass seine Bestimmungen vernünftig sind. An diese Einsicht appellieren etwa die Begründungen von Rechtssätzen, die auf allgemein-menschliche Erfahrungen verweisen ${ }^{12}$ oder spezielle geschichtliche Erfahrungen Israels in Erinnerung rufen, die für seine kulturelle Identität bestimmend sind. ${ }^{13}$ Wenn die Israeliten sich nach Ex 24,3-8 am Sinai zweimal ausdrücklich zur Einhaltung der ihnen von Mose verkündigten und dann noch einmal verlesenen Gebote Gottes verpflichtet haben, wird damit verdeutlicht, dass ihnen das Gesetz nicht aufgenötigt wurde, sondern Inhalt eines "Bundes“ zwischen Jahwe und Israel ist, dem Israel freiwillig und aus Einsicht zugestimmt hat (vgl. Dtn 26,16-19; 28,69). ${ }^{14}$

Ausdrücklich hervorgehoben wird die Einsichtigkeit und Vernünftigkeit des Gesetzes in Dtn 30,11-14: ${ }^{15}$

10 Vgl. v.a. Jes 2,1-5/Mi 4,1-5 sowie Jes 42,1-3; 51,4f.; Fischer, Irmtraud, Tora für Israel Tora für die Völker. Das Konzept des Jesajabuches, SBS 164, Stuttgart 1995.

11 In Dtn 6-11 wird mehrfach auf geschichtliche Erfahrungen Israels hingewiesen, die die Autorităt und Sanktionsgewalt des gottlichen Gesetzgebers deutlich machen (vgl. auch Dtn 4,3f.)

12 Vgl. etwa Ex 22,15f.; 23,8; Dtn 15,18; 16,19; 20,19; 24,13

$13 \mathrm{Vgl}$. etwa Ex 23,9; Dtn 4,15ff.; 5,15; 23,4ff.

14 Diese Pointe der Verbindung von Bund und Gesetz im AT wird deutlich im Vergleich mit der Legitimation des Rechts durch die Autoritat des von den Göttern eingesetzten Königs in Mesopotamien, vgl. z.B. TUAT I, 24f.30f. (Lipit Eschtar); 40ff.75ff. (Hammurapi).

15 Vgl. zur neueren Diskussion über diesen Abschnitt Otto, Eckart, Das Deuteronomium im Pentateuch und Hexateuch. Studien zur Literaturgeschichte von Pentateuch und Hexa-
(11) Denn dieses Gebot (מקוה), das ich dir heute erteile, es ist nicht zu schwierig fur dich und es ist nicht (zu) fern. (12) Es ist nicht im Himmel, sodass man sagen müsste: „Wer steigt für uns in den Himmel und holt es uns, damit wir es hören und befolgen können?" (13) Es ist auch nicht jenseits des Meeres, sodass man sagen musste: „Wer überquert fur uns das Meer und holt es uns, damit wir es hören und befolgen können?" (14) Nein, das Wort ist dir sehr nahe in deinem Mund und in deinem Herzen, sodass du es befolgen kannst.

Das Gesetz ist leicht zugänglich, praktikabel und „deinem Mund und deinem Herzen nahe". Im Kontext ist dieser Abschnitt wohl nicht so zu verstehen, dass die Nähe der Thora zum Herzen der Israeliten das Resultat einer aktiven "Verinnerlichung" und „Beherzigung" des Gesetzes durch Israel ist, wie sie Dtn 6,6ff. und 11,18ff. fordern, oder dass sie eine Konsequenz der in V. 6 den aus dem Exil heimgekehrten Israeliten verheißenen Beschneidung des Herzens durch Jahwe darstellt. ${ }^{16}$ Vielmehr begründet Dtn 30,11-14 den in V. 10 vorangehenden Aufruf an die (exilierten) Israeliten zu einer (erneuten) Thora-Beherzigung mit dem Hinweis darauf, dass das Gesetz der menschlichen Vernunft (dem „Herzen“) nicht widerspricht, sondern ihr entgegenkommt. ${ }^{17}$

\section{III.}

Nicht nur für das Verständnis des Gesetzes benötigt der Mensch Weisheit und Einsicht, sondern auch für seine Anwendung in der Rechtsprechung.

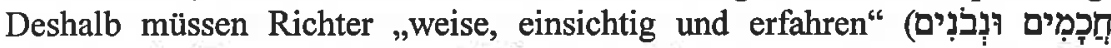
(וידעים sein (Dtn 1,13.15) und dürfen sich nicht bestechen lassen, denn „Bestechung macht die Augen von Weisen blind" (Dtn 16,19). ${ }^{18}$ Ex 18,13-26 formuliert mit etwas anderer Terminologie ganz ähnliche Anforderungen an die Richter, wobei besonders bemerkenswert ist, dass die Rechtsordnung Israels hier auf die Initiative eines Midianiters zurückgeführt wird. In Ex 18

teuch im Lichte des Deuteronomiumrahmens, FAT 30, Tübingen 2000, 153f. mit Anm. 179

16 Vgl. Braulik, Georg, Deuteronomium II. 16,18-34,12, NEB.AT 28, Wuirzburg 1992, 219 „Nach 6,6f ist das Gesetz auswendigzulernen (,Herz') und stăndig zu rezitieren (,Mund'). Wenn Israel sich daran hält, braucht es niemals auf die Suche nach dem Gotteswillen zu gehen " Im vorliegenden Textzusammenhang (Dtn 30,1-14) wird der Abschnitt V. 11-14 nach Braulik dann allerdings durch V. 6 in dem Sinne neu interpretiert, dass Gott selbst den Israeliten das Gesetz in ihr Herz gibt: „Da Gott die Herzen beschneiden wird, ist das Gesetz im Herzen (6), und ,Horen' (...) und ,Handeln' nach ihm (...) sind dem Menschen möglich." $\mathrm{Zu}$ dieser letzteren Deutung vgl. auch Spieckermann, Hermann, Gottes Liebe zu Israel. Studien zur Theologie des Alten Testaments, FAT 33, Tubingen 2001, 171.

17 Es würde wenig Sinn machen, wenn V. 11-14 zur Begrindung des Aufrufs zur ThoraBeherzigung auf einen Sachverhalt verweisen wurde, der erst aus der geforderten Beherzigung der Thora resultiert.

18 Vgl. Braulik, „Weisheit“ (s. Anm. 8), 43ff.60ff. 
wie in Dtn 1 wird den Richtern ein Entscheidungsspielraum eingeräumt, der ihnen eine gewisse Freiheit gegenüber dem Gesetz zugesteht (das in Ex 18 ohnehin noch gar nicht erlassen worden ist). Schwierige und gewichtige Fälle müssen sie allerdings Mose vorlegen.

Dessen Nachfolge übernehmen dann nach Dtn 17,8-13 Priester bzw. Richter am Zentralheiligtum. Ihre Entscheidung, die wechselweise als

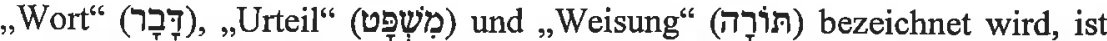
verbindlich und unter Androhung der Todesstrafe zu befolgen. Wie die Priester und Richter zu ihren Entscheidungen kommen, sagt der Text nicht. ${ }^{19}$ Sein Interesse scheint eher darin zu bestehen, Freiräume in der Gesetzesauslegung durch Verfahrensregeln $\mathrm{zu}$ begrenzen, als darin, solche Freiräume $\mathrm{zu}$ eröffnen. Damit zeigt er aber - vielleicht wider Willen -, dass das Gesetz gerade wenn seine Abgeschlossenheit und Unveränderbarkeit betont wird (Dtn 4,2;13,1) - das Leben nicht vollständig und lückenlos regulieren kann.

Neben der Anwendung des Gesetzes in der Rechtsprechung erfordert auch seine Tradierung eine verständige Mitwirkung menschlicher Instanzen. Es muss nicht nur als schriftliches Dokument verwahrt, vervielfältigt und verlesen werden (Dtn 31; 17,18); Eltern müssen es auch ihren Kindern bekannt machen und erklären, damit diese es verstehen und befolgen können (Ex 12,26f.; 13,8; Dtn 6,20-25). Wenn von Josua als dem Nachfolger Moses gesagt wird, er sei vom „Geist der Weisheit" erfüllt gewesen, und die Israeliten hätten ihm gehorcht und das Gesetz Moses befolgt (Dtn 34,9), ${ }^{20}$ ist hier wohl die Weisheit im Blick, deren es bedarf, um das Gesetz so zu vermitteln, dass es dem Volk verständlich und von ihm aus Einsicht eingehalten wird.

Die Hinweise darauf, dass für die Anwendung des Gesetzes in der Rechtsprechung und für seine Tradierung und Propagierung besondere Weisheit erforderlich ist (III.), stehen in einer gewissen Spannung zu der Überzeugung, dass die Gerechtigkeit und Vernünftigkeit des Gesetzes für jedermann unschwer einzusehen ist (II.). Trotzdem halten sie daran fest, dass die besondere Kompetenz, über die Richter oder Gesetzeslehrer verfügen müssen, sich nicht wesentlich unterscheidet von den Kompetenzen, die für andere spezialisierte Tätigkeiten erforderlich sind, oder von der Kompetenz zu einer gerechten und vernünftigen Lebensführung - in all diesen Fällen handelt es sich um ,Weisheit"

$19 \mathrm{Zu}$ vermuten ist die Anwendung divinatorischer Mittel, vgl. Ex 22,7; Lev 24,10ff; Num $5,11 \mathrm{ff}$; $15,32 \mathrm{ff}$.

20 Vgl. dazu Braulik, „Weisheit“ (s. Anm. 8), 63ff.

\section{$I V$.}

Dem scheint nun allerdings Num 15,37-41 zu widersprechen. Hier stehen die Gebote der Thora in schroffem Gegensatz zur eigenen Einsicht und Vernunft der Israeliten:

(37) Und Jahwe sprach zu Mose: (38) Sprich zu den Israeliten und sage ihnen, dass sie sich Quasten an den Zipfeln ihrer Kleider anbringen sollen, sie und ihre Nachkommen, und dass sie an dieser Zipfel-Quaste eine Schnur aus violettem Purpur befestigen sollen. (39) Das soll eure Quaste sein. Wenn ihr sie seht, sollt ihr an alle Gebote (מְְ̦în) Jahwes denken und sie befolgen und nicht eurem Herzen und euren Augen nachforschen, ${ }^{21}$ sodass ihr euch von ihnen zur Unzucht verleiten lasst ${ }^{22}(40)$ - damit ihr an alle meine Gebote denkt und sie befolgt und heilig seid für euren Gott. (41) Ich, Jahwe, bin euer Gott, der ich euch aus dem Land Ägypten gefüht habe, um euer Gott zu werden; ich, Jahwe, bin euer Gott.

Hier kommt eine tiefe Skepsis gegenüber den Möglichkeiten menschlicher Weisheit und Einsicht zum Ausdruck. ${ }^{23}$ Sie verführen die Israeliten geradezu zum Götzendienst. ${ }^{24}$ Das menschliche „Herz" ist ja nicht nur Organ der vernünftigen Überlegung und des Willens, sondern auch der Lust und des Begehrens. ${ }^{25}$ Und die „Augen“" vermitteln dem Menschen nicht nur Einsichten in die Realität, sondern auch Anreize, die seine Begehrlichkeit wecken und ihn zu unvernünftigen und ungerechten Handlungen verleiten können. ${ }^{26}$ Deshalb sollen sich die Israeliten in ihrer Lebensführung nach Num 15 nicht an ihren eigenen Erfahrungen und ihrer eigenen Vernunft orientieren, sondern an den Geboten Jahwes.

Dabei bleibt allerdings unklar, welche Rolle das Herz und die Augen für das Verständnis und die Anwendung der göttlichen Gebote spielen - immerhin müssen die Israeliten ja zumindest ihre Augen gebrauchen, um sich von den Quasten an ihren Gewändern an die Gebote Jahwes erinnern zu lassen. Die in Num 15,32-36 vorangehende Episode suggeriert, dass hier kein wirkliches Problem besteht: Fälle, für die sich im Gesetz keine eindeutigen Regelungen finden, sind nicht etwa durch eigene Überlegung zu lösen; vielmehr ist eine neuerliche göttliche Offenbarung abzuwarten (vgl. Lev 24,10-23). Doch auch diese muss ja wahrgenommen und verstanden werden. Sind das Verständnis und die Befolgung des Gesetzes dann nicht in noch radikalerer Weise durch die Schwächen menschlicher Vernunft und Einsicht gefährdet, als Num $15,37 \mathrm{ff}$. annimmt?

21 Vgl. HALAT (s.v., ad loc.): „nachspüren“ (anders Gesenius - Buhl: „folgen, sich v. etw. verlocken lassen").

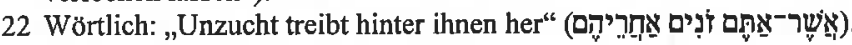

23 Vgl. etwa Spr 16,1ff.; $21,30 f$;; 30,1 ff.

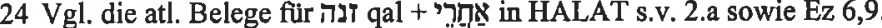

25 Vgl. HALAT und Stolz, Fritz, לב לב לב

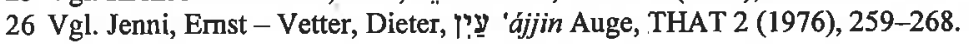


Davon gehen eine Reihe von Aussagen in Dtn 29-32 aus: Herz, Augen und Ohren der Israeliten waren zumindest bis zum Zeitpunkt der Moserede im Deuteronomium gar nicht fähig, aus den Erfahrungen seit der Befreiung aus Ägypten die richtigen handlungsleitenden Konsequenzen zu ziehen (Dtn 29,3). Das Sinnen und Streben ('יצֶ.) der Israeliten lässt Mose schon vor dem Einzug in das verheißene Land erkennen, dass sie das Gesetz nicht befolgen

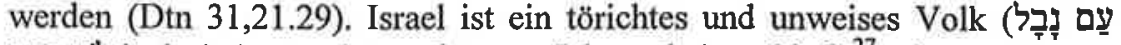

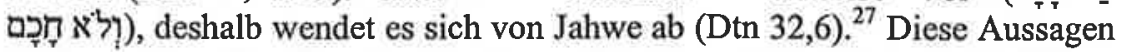
führen das Gesetzesverständnis im Pentateuch (und besonders im Deuteronomium) in eine Aporie: Israel als Volk und die einzelnen Israeliten sind für eine gedeihliche Lebensführung angewiesen auf das von Gott offenbarte Gesetz. Um das Gesetz verstehen, akzeptieren und befolgen zu können, bedürfen sie der Weisheit und Einsicht. Daran aber mangelt es ihnen so sehr, dass sie weder selbst zu einer förderlichen Lebensorientierung in der Lage sind noch die ihnen von Gott im Gesetz angebotene Lebensorientierung annehmen können.

Einen Ausweg aus dieser Aporie zeigt Dtn 30,6 auf: ${ }^{28}$ „Bis heute“ (d.h. bis zur Verkündigung des Deuteronomiums durch Mose) hat Jahwe den Israeliten noch ,kein Herz gegeben, das erkennen kann, keine Augen, die sehen können und keine Ohren, die hören können" (Dtn 29,3). Dereinst aber wird Jahwe „dein Herz und das Herz deiner Nachkommen beschneiden, sodass du Jahwe, deinen Gott, lieben kannst von ganzem Herzen und von ganzer Seele, um deines Lebens willen" (Dtn 30,6). Jahwe selbst muss und wird also die Voraussetzungen schaffen, die das Verständnis und die Befolgung des Gesetzes allererst ermöglichen. Diese Erwartung eines göttlichen Eingriffs ist in Dtn 30,1-10 eng verschränkt mit Aussagen über Israels „Umkehr“ zu Jahwe und Befolgung seines Gesetzes:

- Wenn Israel in der Diaspora zu Jahwe umkehrt (שוכ) und „von ganzem Herzen und von ganzer Seele“ auf sein Wort hört, dann wird Jahwe Israel wieder (שרכ) sammeln und in seine Heimat zurlick bringen (V.1-5)

- Jahwe wird das Herz der Israeliten beschneiden, sodass sie ihn „von ganzem Herzen und von ganzer Seele" lieben werden. Israel wird wieder (שוב) auf das Wort Jahwes horren und seine Gebote halten (V.6-8).

- Jahwe wird Israel im Land Wohlstand schenken und wieder (שוב) Freude an seinem Volk haben, wenn Israel auf sein Wort hört, sein Gesetz befolgt und zu ihm „umkehrt“ (בוש) ,von ganzem Herzen und von ganzer Seele" (V.9f.).

27 Vgl. Gen 6,5; 8,21 und ähnliche Aussagen in den Prophetenschriften (siehe Krüger, Thomas, Das menschliche Herz und die Weisung Gottes. Elemente einer Diskussion uber Möglichkeiten und Grenzen der Tora-Rezeption im Alten Testament, in: Kratz, Reinhard G. u.a. (Hg.), Rezeption und Auslegung im Alten Testament und in seinem Umfeld. Ein Symposion aus Anlass des 60. Geburtstags von Odil Hannes Steck, OBO 153, Freiburg/Schweiz u.a. 1997, 65-92, 81f.)

28 Vgl. Jer 24,7; 31,33; 32,39f.; Ez 11,19; 36,26f. (siehe Krüger, Herz [s. Anm. 27], 82ff.).
Die Umkehr Israels und seine Gesetzesbefolgung, die V. 1-5 und V. 9f. als Bedingung und Voraussetzung seiner Heimkehr aus der Diaspora betrachten, sind nach V. 6-8 ihrerseits erst ermöglicht durch einen Eingriff Jahwes, der Israel dazu befähigt, das Gesetz zu verstehen und zu befolgen. Andererseits schließt aber V. 6-8 narrativ bruchlos an das in V. 1-5 Vorhergehende an, sodass der Eindruck entsteht, die Beschneidung des Herzens finde erst nach der Umkehr und Heimkehr Israels statt. ${ }^{29}$ Diese Spannung legt die Annahme nahe, dass es sich bei V. 6-8 um einen späteren Zusatz handelt. ${ }^{30}$ Sie kann aber zugleich auch als sachgemäßer Hinweis darauf verstanden werden, dass Umkehr und Gesetzesbefolgung Israels zugleich als dessen eigenes Handeln und als Wirken Jahwes begriffen werden müssen. Beides kann voneinander unterschieden, aber nicht zeitlich auseinander gelegt und gegeneinander verrechnet werden. ${ }^{31}$ In diesem Sinne lassen sich dann auch die Ankündigung einer Beschneidung des Herzens durch Jahwe in Dtn 30,6 und die Aufforderung an die Israeliten, ihre Herzen zu beschneiden, in Dtn 10,16 miteinander verbinden. ${ }^{32}$

Wie aber soll man sich die Beschneidung des Herzens durch Jahwe oder durch die Israeliten selbst konkret vorstellen? Die Texte sagen darüber kaum etwas aus, deuten aber im jeweiligen Kontext (Dtn 10,12-22; 30,1-10) mindestens an, dass dabei das Bemühen um ein Verständnis und eine Befolgung des Gesetzes sowie das Lernen aus guten wie schlechten geschichtlichen Erfahrungen ${ }^{33}$ eine Rolle spielen. So scheint es am Ende auch (!) das Gesetz selbst zu sein, das dem Menschen die Weisheit und Einsicht vermittelt, deren er bedarf, um das Gesetz als vernünftig zu begreifen und in die Tat umsetzen zu können. Als Wirken Gottes ist diese Transformation der Vernunft durch das Gesetz jedoch für den Menschen nicht verfügbar und nicht erzwingbar. Vor allem aber hat die „Beherzigung" des Gesetzes nicht wie in Num 15 das Ziel, menschliche Erfahrung („Augen“) und Überlegung („Herz") auszuschalten, sondern diese von hartnäckiger (vgl. Dtn 10,16!) Verblendung und Selbsttäuschung zu befreien.

29 Vgl. Braulik, Deuteronomium II (s. Anm. 16), 218; Rose, Martin, 5. Mose, 2. 5. Mose $1-$ 11 und 26-34, Rahmenstlucke und Gesetzeskorpus, ZBK.AT 5/2, Zürich 1994, $555 f$.

$30 \mathrm{Vgl}$. Krliger, Herz (s. Anm. 27), 79f.

$31 \mathrm{Vgl}$. Phil 2,12f. Die Spannung resultiert daraus, dass (und führt nicht in einen logischen Widerspruch, weil) es hier um ein Handeln Gottes geht, das menschliche Handlungsfreiheit (wieder) herstellt.

32 Vgl. Jer 4,6 und Ez 18,31 neben den oben (Anm. 28) genannten Aussagen bei Jer und Ez.

33 Vgl. Dtn 10,15(Erwählung der Văter durch Jahwe).19(Aufenthalt Israels in Ägypten).21 (erfahrene Wundertaten Jahwes).22(Vermehrung Israels in Ägypten); 30,1(Eintreffen von Segen und Fluch; Diaspora). 
$V$

Die Aussagen über die Verblendung Israels und ihre Beseitigung durch Jahwe in Dtn 29-32 implizieren, dass die Bedingungen des Verstehens und der Befolgung des Gesetzes sich im Laufe der Geschichte verändern. Dies wird in den Texten freilich nur (durch einige späte Zusätze) angedeutet. Einer geschichtlichen Relativierung des Gesetzes selbst stehen im Deuteronomium sowohl die sog. „Kanonformel“ (Dtn 4,2; 13,1) entgegen als auch die Überzeugung, dass dieselbe Thora, die Mose einst vor dem Einzug Israels ins verheißene Land verkündete, auch für die Jahrhunderte später exilierten Israeliten verbindlich ist (Dtn 30,2). Daneben gibt es im Pentateuch allerdings auch einige Hinweise, die eine geschichtliche Entwicklung des Gesetzes zumindest möglich erscheinen lassen.

Die Entstehung der mosaischen Thora wird im Pentateuch als ein längerer geschichtlicher Prozess dargestellt. Jahwe übermittelt Mose das Gesetz auf dem Sinai in mehreren Etappen (vgl. Ex 20,21; 24,12; 34,1ff.). Danach empfängt Mose weitere Bestimmungen im Zeltheiligtum (Lev 1,1; Num 1,1), zum Teil ad hoc angesichts konkreter Problemfälle (Lev 24,10ff; Num 15,32ff.; 36). Im Deuteronomium verkündet Mose nach vierzigjährigem Aufenthalt in der Wüste, bei dem nahezu die gesamte Exodusgeneration gestorben ist, noch einmal die ganze Thora einer neuen Generation von Israeliten. Dabei wird in Dtn 5 wie in Ex 20 der Dekalog als grundlegende ethische Orientierung den ausführlichen und detaillierten Rechtsbestimmungen vorangestellt.

In diesen Rechtsbestimmungen gibt es nun aber zwischen Ex-Num und Dtn Differenzen, die auch für „vorkritische“ Leser kaum zu übersehen sind. ${ }^{34}$ Die in der „Kanonformel" fixierte Abgeschlossenheit und Unveränderlichkeit des Gesetzes kann so mit Blick auf den vorliegenden Pentateuch als Ganzes kaum im Sinne einer buchstäblichen Fixierung aller Einzelheiten verstanden werden. Vielmehr enthält der Pentateuch die für eine ethische und rechtliche Orientierung notwendigen „Lehren" und „Weisungen“ (תרְi), die Entscheidungen in konkreten Fällen ermöglichen, solche Entscheidungen aber auch immer wieder erfordern (s.o. III.). ${ }^{35}$ In seiner vorliegenden literarischen

34 Vgl. z.B. die z.T. widersprlichlichen Bestimmungen über das Passa in Ex 12 und Dtn 16 oder über Schuldenerlass und Sklavenfreilassung in Ex 21; Lev 25 und Dtn 15.

35 Dem entspricht etwa das Thora-Verständnis der Qumran-Gemeinschaft. Sie hatte offenbar in ihrer „Frlihphase ... ein noch recht fließendes, dynamisches Torahverstandnis mit der Möglichkeit neuer Torah-Anweisung durch den ,Lehrer“", wăhrend sich in den „späteren Perioden eine Beschränkung auf die Anwendung und interpretierende Adaptierung der vorhandenen Torah-Traditionen" zeigt. „Diese letzteren lagen in einem viel weiteren Umfang schriftlich fixiert vor, als man noch vor wenigen Jahren ahnen konnte, jedenfalls steht fest, daß es geschriebene Torah auch außerhalb des Pentateuchs gab und daß für die Qualităt einer Einzelregelung als ,Torah" nicht maßgeblich war, ob sie im Pentateuch vorhanden oder von dorther begründet werden konnte ... Das Phänomen einer über den
Gestalt stellt der Pentateuch m.a.W. keinen "Codex" dar, sondern ein "Rechts(lehr)buch“" ${ }^{36}$ Als solches will er seinen Lesern ethische und rechtliche Kompetenz - d.h. in der Sprache des AT: „Weisheit" - vermitteln.

$V I$.

Die skizzierten Beobachtungen deuten darauf hin, dass die Diskussion über das Verhältnis von Weisheit und Gesetz, die im 2. Jh. v.Chr. bei Sirach und Baruch greifbar wird, im Pentateuch selbst bereits angelegt und vorbereitet ist, auch wenn dabei nicht immer der Terminus "Weisheit" verwendet wird. Das ist von der Sache her auch nicht anders zu erwarten, geht es doch im Gesetz wie in der Weisheit - mit je verschiedener Akzentsetzung - um die Regulierung und Orientierung der Lebensführung des Menschen.

Der Pentateuch bringt immer wieder die grundlegende Überzeugung zum Ausdruck, dass es weise und vernüntig ist, das Gesetz zu befolgen - weil das Gesetz dem Menschen als weise und vernünftig einsichtig ist. Gesetz und Weisheit sind dementsprechend eng miteinander verbunden. Beide sind dem Menschen aber nicht selbstverständlich gegeben. Die Völker erkennen die Weisheit und Gerechtigkeit des Gesetzes Israels, waren aber offenbar selbst nicht dazu in der Lage, sich ebenso weise und gerechte Gesetze zu geben (Dtn 4). Und Israel verfügte zum Zeitpunkt der Gesetzesverkündigung (noch) nicht über die nötige Weisheit, um die Gerechtigkeit und Vernünftigkeit des Gesetzes einsehen und es befolgen zu können (Dtn 29f.). Gesetz und Weisheit sind also gleichermaßen Gaben Gottes.

Wenn das Gesetz im Pentateuch als Offenbarung Gottes verstanden wird, wird damit sowohl seine Einsichtigkeit als auch seine Unerschwinglichkeit für die menschliche Vernunft festgehalten. Das Gesetz ist eine - durch Tradition vermittelte - Vorgabe für die menschliche Lebensführung und Lebensorientierung, die immer wieder neu verstanden, interpretiert und angewendet

Pentateuch hinausgehenden Torahtradition ist nicht erst durch die Qumrantexte belegt", wie ein Vergleich von Neh 10,35 mit dem Pentateuch einerseits und mit Jub 21,12-14 und 11Q19 Kol. 23-25 andererseits zeigt (Maier, Johann, Die Qumran-Essener. Die Texte vom Toten Meer, 3. Einfiihrung, Zeitrechnung, Register und Bibliographie, UTB 1916, München u.a. 1996, 13f.).

36 Während Codices „präskriptiv Rechtsentscheide normieren wollen“, haben Rechtsblicher ,eher eine Funktion in der Schreiber- und Beamtenausbildung der Schreiberschulen und wollen in den Rechtsentscheid einüben" (Otto, Eckart, Gesetz I. AT, in: Bauer, Johannes B. u.a. [Hg.], Bibeltheologisches Wörterbuch, Graz u.a. ${ }^{4}$ 1994, 231-233, 232). Vgl. Otto, Eckart, Die Bedeutung der altorientalischen Rechtsgeschichte für das Verstăndnis des Alten Testaments, ZThK 88 (1991), 139-168; ders., Kodifizierung und Kanonisierung von Rechtssätzen in Keilschriften und biblischen Rechtssammlungen, in: Lévy, Edmond (Hg.), La codification des lois dans l'antiquité. Actes du colloque de Strasbourg 27-29 novembre 1997, Travaux du Centre de Recherche sur le Proche-Orient et la Grèce Antiques 16, Paris 2000, 77-124. 
werden muss - auch im ,interkulturellen" Dialog (der wenigstens ansatzweise in Dtn 4 in den Blick kommt). Der Pentateuch betont dabei stark den Vorgabecharakter des Gesetzes und fordert zum Festhalten am Gesetz als lebensorientierender Tradition auf (in problematischer Zuspitzung in Num 15; vgl. Bar 3f.). Recht und Notwendigkeit einer kritischen Weiterentwicklung des Gesetzes werden in anderen Traditionsbereichen des Alten Testaments (v.a. Prophetie und Weisheit) - und dann im Neuen Testament - deutlicher erkennbar. Bei genauerem Zusehen lassen sich aber schon im Pentateuch selbst Ansätze zu einem „liberaleren“ Gesetzesverständnis entdecken, wie es dann etwa Jesus Sirach - für seine Zeit - durch die Identifikation von Weisheit und Gesetz in Sir 24 zu formulieren versucht hat.

\section{Die sieben Säulen der Weisheit im Buch Deuteronomium}

Georg Braulik, O.S.B.

\section{Die literarische Architektonik als Vergleichspunkt}

Das Haus, das sich Frau Weisheit nach Spr 9,1 gebaut hat, wird von sieben, aus Stein gehauenen Säulen getragen. In dieses Sieben-Säulen-Gebäude lädt sie die "Unwissenden" zum Gastmahl der Lehre, damit ihr Leben gelingt $(9,2-6)$. Über die architektonische bzw. metaphorische Deutung der sieben Säulen wurde schon viel diskutiert. ${ }^{1}$ Trotz des archäologischen Realienhintergrunds interpretiert man sie heute wohl am plausibelsten als Anspielung auf die mit sieben Überschriften versehenen Teile des Sprichwörterbuches. ${ }^{2}$ Die Weisheit bittet also die Unerfahrenen zu Bankett und Studium in den Proverbien-„Palast" als ihr Lehrgebäude. Das Motiv der sieben Säulen der Weisheit ist im Alten Testament singulär. Wenn der Titel dieses Beitrags es trotzdem mit dem Deuteronomium verbindet, dann nicht, um eine analoge Bauweise des Buches Deuteronomium, eine Siebenteilung oder gar eine explizit siebenfältige Gliederung durch Überschriften bzw. Redeeinleitungen, zu suggerieren. ${ }^{3}$ Ebenso wenig geht es um eine „weisheitliche“ Prägung des Deuteronomiums. ${ }^{4}$ Der Vergleichspunkt liegt vielmehr in der Siebenzahl als

1 Die wichtigsten Erklärungsmodelle resumieren z.B. Maier, Christl, Die ,fremde Frau“ in Proverbien 1-9. Eine exegetische und sozialgeschichtliche Studie, OBO 144, Freiburg/Schweiz u.a. 1995, 231-233, und Baumann, Gerlinde, Die Weisheitsgestalt in Proverbien 1-9, FAT 16, Tübingen 1996, 202-207.

2 Baumann, Weisheitsgestalt (s. Anm. 1), 207-209.

3 Z.B. gegen Christensen, Duane L., Deuteronomy 1:1-21:9, revised, WBC 6A, Nashville 2001 , xciiif. Eine solche Siebenteilung dürfte im Pentateuch das Buch Levitikus aufweisen. Zu seiner siebengliedrigen Komposition, die durch strukturelevante Einleitungsformeln der Gottesreden angezeigt wird, und zur theologischen Programmatik dieser Einteilung siehe Zenger, Erich, Das Buch Levitikus als Teiltext der Tora/des Pentateuch. Eine synchrone Lekture mit kanonischer Perspektive, in: Fabry, Heinz-Josef u.a. (Hg.), Levitikus als Buch, BBB 119, Berlin u.a. 1999, 47-83, 65-76. Zu Siebenergruppierungen von Schlüsselwörtern und siebenteiligen Mikro- und Makrostrukturen in diesem Buch siehe Warning, Wilfried, Literary Artistry in Leviticus, BIS 35, Leiden u.a. 1999, 27-29. 133-135:170f. Analoge Phänomene dürten auch im Amosbuch vorliegen - vgl. Möller, Karl, A Prophet in Debate. The Rhetoric of Persuasion in the Book of Amos, JSOT.S 372, Sheffield 2003, 83-86.

4 Sie wird v.a. von Weinfeld, Moshe, Deuteronomy and the Deuteronomic School, Oxford $1972(=21990), 244-319$, vertreten. Dass die an Thematik, Terminologie und Stil beob- 